\section{Is maintenance of LTP presynaptic?}

SIR - Two recent suggestions ${ }^{1,2}$ that the synaptic enhancement underlying long-term potentiation (LTP) in neurons is maintained by presynaptic mechanisms are based on the fact that, given a binomial model of transmitter release at a single connection, changes in a quantitative measure of the variability of the postsynaptic responses (coefficient of variation) should be indicative of a presynaptic locus for the modifications. In both, the assumption was that in the preparation of hippocampal slices a single afferent was reliably stimulated by 'minimal extracellular stimulation', so that all failures occurred at the release step. In this case, one presynaptic neuron projecting to the target cell must be stimulated consistently while no other inputs are activated, even intermittently. Although

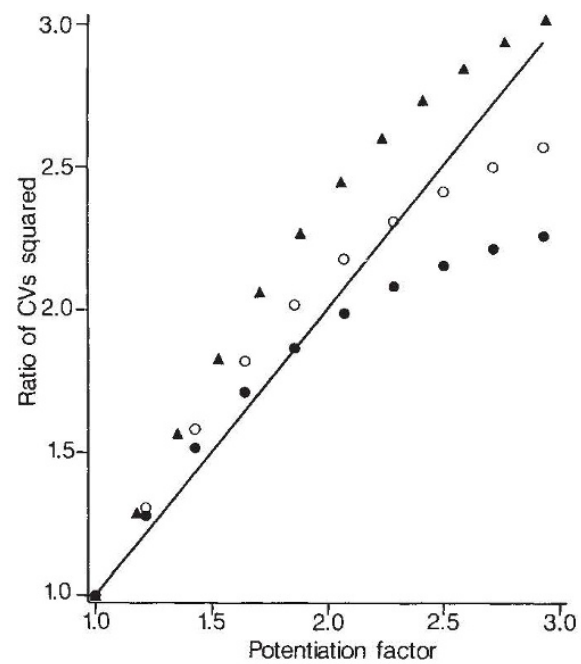

Relation between changes in synaptic efficacy (abcissa) and the reduction in coefficients of variation (CV-ordinates) after LTP, assuming an increase of quantum size alone. The presynaptic parameters used were, $n=9, p=0.6$ for $\boldsymbol{\Delta}$ and 0 , and $n=7$, $p=0.6$ for - while the probability that individual cells were excited by the stimulus ranged, in each series, from 100 to $10 \%$. Solid line, data interpreted as representing a presynaptic modification ${ }^{2}$. $\mathbf{\Delta}, N=6, n p=5.4$ $\mathrm{O}, N=3, n p=5.4, \ominus, N=3, n p=4.2$.

it is understood that the coefficient of variation method is a powerful but indirect tool (see refs 3,4 ), it seems reasonable to explore the alternative that the extracellular stimulus might excite several afferents, with there being intermittent failures of impulse initiation in some of them.

If the analysis developed by Bekkers and Stevens ${ }^{2}$ for multiple inputs is expanded in this way, one can find similar results (potentiation equalling the ratio of the coefficients of variation squared) when quantal size, $q$, is the only parameter modified. This occurs if $q$ at individual connections is enhanced as a nonlinear function of the fraction of time each afferent cell is activated, a formulation consistent with a hebbian model for potentiation. This is illustrated in the figure for which it was assumed that several $(N)$ cells were involved, all with the same initial values of $q, n$ and $p$, the last two being the number of release sites $^{3}$ and release probability, respectively. The predictions are similar to the experimental observations ${ }^{1,2}$, with the agreement covering a broader range if the neurons have relatively large $n p$ products or more cells are involved. Also, assuming multiple afferents, one finds a wide range of parameter sets for which Poisson fits of evoked histograms appear, as in slice experiments ${ }^{2}$, superior to simple binomials. In these situations, failures at individual connections would be hidden.

Thus, in the case of extracellular stimulation, the coefficient of variation does not reliably distinguish between pre- and postsynaptic sites of modification. The notion that maintenance of LTP has a major presynaptic locus even though the process is initiated postsynaptically is inherently attractive, as it emphasizes the unity of a synapse. It is to be hoped that this issue can be resolved by a more direct structural and quantal analysis of LTP at connected cell pairs, such as the preliminary data referred to in ref. 1 , although this approach has met with limited success in the central nervous system, mainly because of the difficulty in resolving quanta ${ }^{3,4}$. (A fuller version of our arguments will be submitted for publication elsewhere.)

HENRI KORN

Carola Fassnacht

Laboratoire de Neurobiologie

Cellulaire,

Département des Biotechnologies, INSERM U261,

Institut Pasteur,

75724 Paris Cedex 15, France

Neurobiology Laboratory,

Donald S. FABER

Department of Physiology,

School of Medicine, State University of New York,

Buffalo, New York 14214, USA

MALINOW AND TSIEN REPLY - Korn et al. use a detailed model to point out a limitation in analysis of hippocampal synaptic transmission ${ }^{1,2}$ based on the coefficient of variation $(\mathrm{CV})^{5}$. This assumption, already stated explicitly in our paper ${ }^{1}$, is that all synapses are homogeneous with respect to postsynaptic responsivity during a given epoch. CV analysis is analogous in this respect to noise analysis of ion-channel properties: both approaches give an over-view of statistical properties, but they require assumptions for quantitative estimates of unitary parameters. Channel noise analysis is often followed up by single channel recordings. Similarly, CV analysis can be complemented by other means for localizing changes in synaptic efficacy: (1) analysis of failures of transmission ${ }^{1,2,6,7},(2)$ measurement of quantal spacing $^{8}$, (3) analysis of the amplitude and frequency of miniature excitatory postsynaptic currents ${ }^{9,10}$, (4) assays or postsynaptic responsiveness by local application of transmitter ${ }^{10-12}$. Clearly, several different approaches are desirable when studying LTP.

These additional approaches are not addressed by Korn et al. The analysis of synaptic failures is one relevant case. Their model predicts no change in failures, clearly contradicting published observations in conventional hippocampal slices with either minimal extracellular stimulation ${ }^{1,2,7}$ or intracellular stimulation ${ }^{1,6}$, or in cultured hippocampal neutrons. In each of these systems, the proportion of failures decreases sharply during LTP.

The model proposed by Korn et al. requires multiple afferent pathways with varying degrees of excitation failure. These complications were addressed in cell-pair experiments in hippocampal slices; a single presynaptic cell was reliably stimulated intracellularly during whole-cell recording from a post-synaptic cell (refs 1, 6, 7 and 13). In each of four pairs of synaptically connected cells, we saw increases in $\mathrm{CV}^{-2}$, a decrease in failures and a shift in the amplitude histogram from a left-skewed form to a more symmetrical distribution (ref. 1, 6, 7, 13). These results, similar to those obtained with extracellular stimulation, provide evidence of presynaptic modifications during LTP, without ruling out possible additional postsynaptic changes.

\section{Department of Physiology and}

RoBerto MaLinow

Biophysics,

University of lowa,

lowa City, lowa 52242, USA

Richard W. TSIEN

Department of Molecular and Cellular Physiology,

Beckman Center,

Stanford University Medical Center, Stanford, California 94305, USA

1. Malinow, R. \& Tsien, R. W. Nature 346, 177-180 (1990).

2. Bekkers, J. M. \& Stevens, C. F. Nature 346, 724-728 (1990).

3. Korn, H. \& Faber, D. S. in Synaptic Function (eds Edelman, G., Gall. W. E. \& Cowan, M. 37-108 (Wiley, New York, 1987).

4. Redman, S. J. Physiol. Rev. 70, 165-198 (1990).

5. del Castillo, J. \& Katz, B. J. Physiol. 124, 560-573(1954).

6. Malinow, R. \& Tsien, R. W. Soc. neurosci. Abs.16, 145 (1990).

7. Tsien, R. W. \& Malinow, R. Cold Spring Harb. Symp. quant Biol. 55, 147-159 (1990).

8. Larkman et al. Nature 350, 344-347 (1991).

9. Finch, D. M. \& Jackson, M. B. Brian Res. 518, 269-273 (1991).

10. Malgaroli, A. \& Tsien, R. W. Biophys. J. 59, 19a (1991).

11. Davies, S. N. et al. Nature 330, 500 (1989).

12. Zilberter, U. I. et al. Neutron 5, 597-602 (1990),

13. Malinow, R. Science (in the press).

\section{Scientific Correspondence}

Scientific Correspondence is intended to provide a forum in which readers may raise points of a scientific character. They need not arise out of anything published in Nature. In any case, priority will be given to letters of fewer than 500 words and five references. 